\title{
Practical Analysis Vertical Handover Decision (VHD) Algorithm for WIMAX and WLAN
}

\author{
S.V. Surwase \\ G.H.Raisoni COEM, Pune
}

\author{
S.S. Sambare \\ PCCOE, Pune
}

\begin{abstract}
Recently the study of efficient vertical handover decision (VHD) algorithm for heterogeneous wireless networks using FR-HMM method which is called as Fuzzy Rule Based Hidden Markov Model (FR-HMM) is presented. In this paper our aim to investigate the performance of this proposed approach under various network conditions the metrics we computed are based on different kinds of applications like text messages, audio and video. For the performance evaluation we basically focused on performance metrics such as average throughput, packet delivery ratio, energy consumption, handover delay, authentication delay etc. The comparison is done among different kinds of application used for communication. Rest of paper is addressing the same proposed mathematic model, literature review and introduction from our previous publications and studies.
\end{abstract}

\section{Keywords}

Vertical Handover, Horizontal Handover, WLAN, GSM, WIMAX, Fuzzy Rules, HMM, Handover delay, performance estimation.

\section{INTRODUCTION}

Heterogeneous Wireless Networks has various access technologies, overlapping coverage and network architecture, protocols for transport, routing and mobility management. Also various operator offer different service demands from mobile user (voice, video, multimedia, text etc) in the market. Because of these variations, when the mobile user moves there is a need to handover the communication channel from one network to another network by considering its features and user requirements [12]. Channel handover between two various networks has been done by vertical handoff. In during handover there is require to decide and choose the best network as mentioned above. So the Vertical Handoff Decision Making is the important research issue [13].

The emergence of several wireless technologies supporting high data rate, multimedia services and coverage, smart mobile terminals with interoperable air interfaces and flexible software component, and the IP based applications produced anytime, anywhere, any type service connectivity platforms for mobile users. Global wireless connectivity is aimed by the (4G) fourth generation wireless systems [1] [2]. Global roaming and high data rate services elevated $4 \mathrm{G}$ service from the former versions of wireless networks [3] [4]. The design goal of the $4 \mathrm{G}$ systems is to provide seamless movement of mobile terminals across the heterogeneous networks by offering continuity of services while maintaining quality of service. Extremely conciliatory and adaptive convergence of several mobile terminals or network technologies backing built-in potentiality for seamless wireless access drives the architectural goals of $4 \mathrm{G}$ systems. It is also important to realize that the arrival and deployment of more wireless technologies offering versatile services can add in the complexity of hand off process (refer to TABLE I).

Tracking the location of the mobile subscribers, are allowing continuity of calls and the other services is the objective of mobility management. Mobility management is the combination of location management and handoff management Change of the point of contact (Base station) while maintaining continuity of services of the mobile terminal during its roaming, is ensured by handoff management [1]. The events that influence handoff management are mobility scenarios and network conditions and user preferences, network selection strategies (handoff decision techniques) for the selection of best network and execution protocols. Every mobility scenario falls into both intra-system and inter-system roaming. Horizontal handoff takes place in intra-system roaming when a mobile terminal departs the regulated realm of one access router and come into the regulated realm of another access router within the same network [10] [11]. Whereas in inter-system roaming, vertical handoff takes place when a mobile terminal moves in between various network technologies are suitable for connectivity reasons depending upon the type and quality of service demanded by the mobile user. The challenge of vertical handoff management is Seamless network switching. Evaluation of received signal strength (RSS) will be insufficient for making only for the vertical handoff decision. Extra parameters like as network conditions, service type, network coverage and cost, power consumption, and user preferences should be taken in to consideration [5]. Finding is the right time for handoff to happen is very crucial for the handovers. Handoff mechanisms can be controlled in 2 ways, either network controlled and mobile terminal controlled mechanisms. Network controlled handover policies are cannot be determine the right time for handoff to take place because they cannot have the latest information of the current circumstances of the mobile terminal. The network controlled mechanisms will not be suitable for execution of vertical handovers because a network cannot be aware of the characteristics of all other networks. Mobile controlled handoff decision schemes will be optimal for vertical handovers since a mobile terminal knows better of its current circumstances.

In this paper we are presenting the new approach for VHD which is based on fuzzy rules and HMM for taking the accurate decision for vertical handover. In next section II we are presenting the literature survey over the various methods those VHD techniques. In section III, the proposed system algorithm and design is presented. In section IV, the proposed approach and its system block diagram is depicted. In section $\mathrm{V}$, the main aim of this paper is depicted with different network results using this proposed approach. Finally conclusion and future work is predicted in section VI. 


\section{REVIEW OF LITERATURE}

In this section we are presenting the different methods for vertical handover decision

In [2], Authors presented an architectural solution based on an agent who determines the target network as the user profile and application. Any terminal that contains the agent platform can access the service providers MAP (Multi Access Provider). The disadvantage of this solution is that it requests more entities and interfaces to provide for user, network and operator.

In [3], this provides a functional interface NIU (Network Interworks Unit) between the SGSN and WLAN to address specifics of the latter. The management of vertical handover in the IP model increases the cost of signaling of different networks and also the latency of the handover. The need to decrease the probability of unnecessary handovers is essential. In [4], author presented a proactive decision handover. It consists of forcing handover before interrupting the link with the previous network. Algorithm of [4] permits to skip dysfunction of a link during the handover, to reduce packet loss and to ensure seamless mobility. The disadvantage of this approach is the risk of reducing the flow caused by the early handover execution which assures the degradation of QoS parameters.

In [5], author suggests to introduce three entities: HoIG (Handover Information Gathering) gathering all the information about the network and the terminal through steps to identify the need for Handover and apply it. HoD (Handover Decision): determines if there is a need to initiate a handover and how to select the best network based on criteria. HoE (Handover Execution connects with the selected target. The handover process is fully controlled by mobile. Execution of Process Analytic Handover is to take the decision of handover. The use of 'scoring criteria': defines the importance of each objective. Priorities have been given by the user. In the 'Network scoring' the available networks are evaluated and compared according to the objectives for each network available, a score is assigned according to user's preferences.

In [6], authors presented the utility-based network selection scheme for HWN, where the price mechanism acting as a lever systems guides users to select the most efficient network or controls the allocation of network resources. Simulation results display that the proposed scheme can achieve more total utilities than traditional schemes for the whole networks. The proposed system can also balance traffic load between different networks and effectively avoid network congestion while still guarantees QoS for real-time users.

In [7], the authors presented the approach for network selection scheme for the integration of UMTS and WLAN. Analytic hierarchy process (AHP) is applied to decide the relative weights of evaluative criteria set according to user preferences or service applications, or grey relational analysis (GRA) is adopted to rank the network alternatives.

In [8], the authors of this paper presented the utility-based algorithm that accounts for user time constraints are estimates complete file delivery time (for each available network) and selects the most promising access network based on consumer surplus (CS) difference.

In [9], presented the quality of network $i$ at a certain time considered as a function of the available bandwidth it can be offer $(\mathrm{Bi})$, the timeliness value $(\mathrm{Ti})$, and error rate value is (
Ei). $Q i=f(B i, T i, E i)$.

All these methods presented so far having their limitations in terms of performance metrics which needs to be rectified further by presented the improved new method.

\section{PROPOSED DESIGN AND ALGORITHM 3.1 Problem Formulation \\ Output Set:}

Performance evaluation metrics for VHD algorithms are NP hard problem.

$\square$ Handover delay: It refers to the duration in between the initiation and the completion of the handover process. Handover delay is related to the complexity of the VHD process, and the reduction of the handover delay is especially important and main for delay-sensitive voice or multimedia sessions.

Number of handovers: Reducing the number of handovers is usually preferred as for the frequent handovers would cause wastage of network resources.

Handover failure probability: A handover failure occurs when the handover is initiated but the target network does not have sufficient for resources to complete it, or when the mobile terminal moves out of the coverage of the target network before the process is finalized. In the former case, the handover failure probability is related to the channel availability of the target network, while in the latter case it is related to mobility of the user [3].

Throughput: The throughput refers to the data rate has been delivered to the mobile terminals on the network. Handover to a network candidate with larger throughput is usually desirable.

\section{NP-Hard Problem}

Finding the optimal BS from the network for Vertical handover is NP-complete problem:

We are using the HMM model for selection of optimal BS.

In its discrete form, a hidden Markov process can be visualized as a generalization of the Urn problem: In a room that is not visible to an observer there is a genie. The room contains urns $\mathrm{X} 1, \mathrm{X} 2, \mathrm{X} 3 \ldots \mathrm{Xn}$. each of which contains a known mix of balls, each ball labeled $y 1, y 2, y 3, \ldots$. The genie are chooses from an urn in that room and randomly draws a ball from that urn. It then puts the ball onto a conveyor belt, where the observer can observe the sequence of the balls but not the sequence of urns from which they are drawn. The genie has some procedure to choose urns; the choice of the urn for the n-th ball depends on only upon a random number and the choice of the urn for the $(n-1)$-th ball. The choices of urn do not directly depend on the urns but chosen before this single previous urn; therefore, this is called a Markov process. .

\subsection{Proposed Problem Solution and Algorithm}

In our proposed handover method we are going to consider WIMAX, WLAN networks for practical analysis of proposed VHD algorithm. The proposed VHD algorithm is based on fuzzy logic and HMM model. In this section we are presenting the detailed architecture of proposed approach.

Following is the basic algorithm steps for proposed VHD. 


\section{VHD Algorithm:}

Step 1: Currently some application is running on mobile.

Step 2: If RSS (Received Signal Strength) is less than RSS threshold then Initialize handover else keep on monitoring RSS for above condition.

Step 3: In handover process collect 1.RSS 2.Data rate 3.Coverage area 4.Bandwidth etc. information of all available networks.

Step 4: According to current user application requirement scores are calculated for each network using fuzzy logic.

Step 5: Check if highest scored network is congested then handover to next high score network else handover to the same network.

Figure 2 below showing the overall flowchart for proposed VHD which is based on fuzzy rules and HMM.

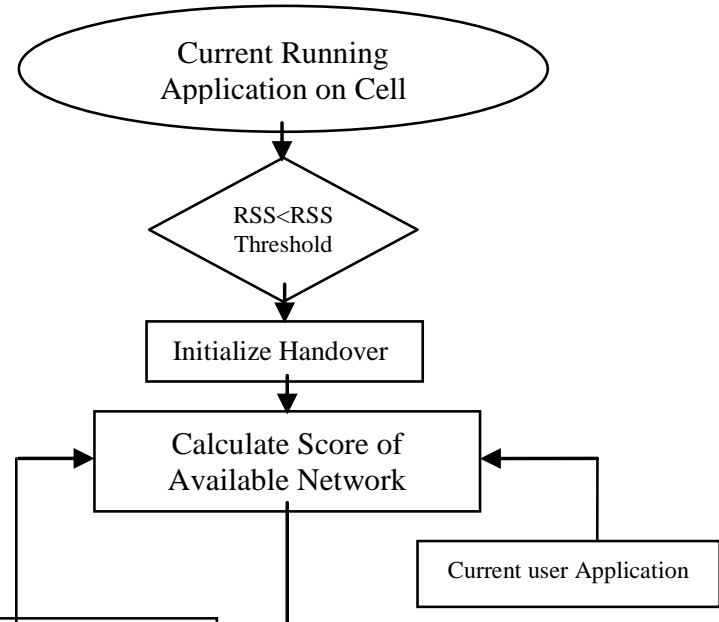

\section{Collect}

- RSS

- Bandwidth

- Data Rate

- Coverage of validate Network

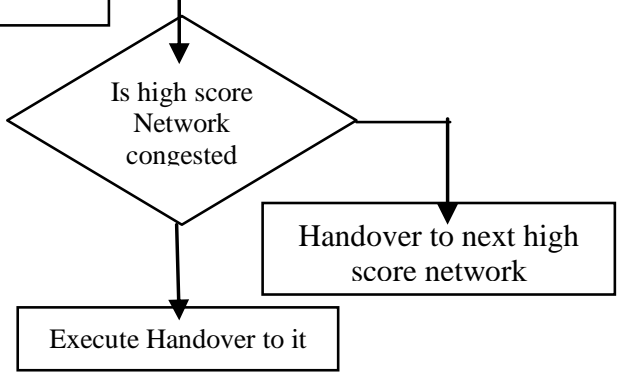

Figure 1: Proposed System Architecture

Fuzzy logic is a process of decision making based on input membership functions and a group of fuzzy rules, like the human brain, which simulates the interpretation of uncertain sensory information. Here, fuzzy logic is applied in order to select the most appropriate network from the list of networks that are available from the scanning phase, which is handled by the MN. Normally, the MN does not know which AP will be a good partner to perform a handover, and it can just depend on the quality of the current and available link. In addition, sometimes, the MN will perform unneeded handovers during its roaming. In other words, the handover obtained by incorrect decision-making will cause overhead signaling and increase the handover latency time. Therefore, fuzzy logic is the answer to this uncertain type of problem.

In our proposed fuzzy logic system, the information gathered (RSS, bandwidth, network coverage, Data rate) depending on their availability are fed into a fuzzifier in which are converted into fuzzy sets. A fuzzy set contains varying degree of memberships in the sets. The membership values are obtained by mapping the values retrieved for the particular variable into a membership function. Figure 2 gives membership functions of the input fuzzy variables.

- The input fuzzy variable "RSS" has three fuzzy sets: weak, normal, and strong.

- The input fuzzy variable "bandwidth" has three fuzzy sets: low, normal, and high.

- The input fuzzy variable "network coverage" has three fuzzy sets: bad, normal, and good.

- The input fuzzy variable "Data rate" has three fuzzy sets: less, normal, and more.

These inputs are chosen answering specific needs related to different scenarios. RSS indicates the current radio link quality and acts as a pretreatment that helps to decide whether to trigger the handover. The bandwidth is different from a network to another (e.g., 3G has lower bandwidth compared to WLAN).

\section{HMM Prediction Model:}

In the proposed work, a HMM based prediction model is executed by each BS or AP in the network. Let us assume that each BS holds the list of their location and other BSs. All of the user movements are in the hidden states. The observed states are obtained from the received signal strength (RSS) values are sent by every mobile node. The BS in which the mobile is currently residing is in the home BS. The home BS predicts the list of possible BSs in the movement of a direction of the mobile station.

\section{Selection of Optimum BS}

The home BS sends a request to each predicted $\mathrm{BSi}$, where $\mathrm{i}=1,2 \ldots \mathrm{v}$. On receiving the request $\mathrm{BSi}$ need to calculate in the Gain function.

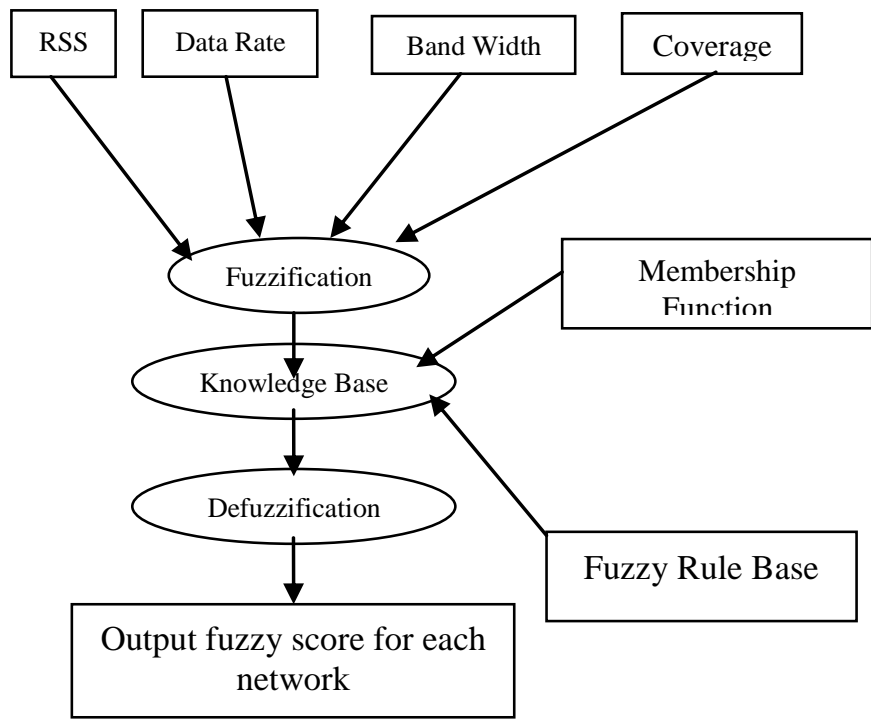

Figure 2: Commutation of Fuzzy Score based network input parameters 
The Gain value is calculated by measuring the normalized values of load and RSS and the power consumed. Gain function is $\mathrm{Gi}=\mathrm{f}(\mathrm{L}, \mathrm{P}, \mathrm{RSS})$ The Gain function is calculated by using the Simple Additive Weight (SAW) algorithm. The Gain functions are calculated only for BSs. The BSs send the calculated Gain functions back to the home BS. The home BS arranges the received weight values in their ascending order and selects from the BS with minimum weight value. The detail of the selected BS is sent to the mobile station so that the $\mathrm{MN}$ can move to that particular BS.

\section{HMM Pseudo Code}

BS [] // Single BS array

ABS [] // All BS's information

tot_bs; Total number Bs

Step 1: for $(i=0 ; i<=$ tot_bs; $i++)$

$$
\begin{aligned}
& \text { BS = ABS [i]; } \\
& \text { Send_Req (i); }
\end{aligned}
$$

Step 2: BS Send request to each other BS, as in above step 1.

Step 3: Inside Send_Req function, gain function calculated.

Send_Req function returns gain_val.

Step 4: Optimal BS section for VHD.

Select (BS) that having less gain value, called opt_bs

Step 5: MN moves to opt_bs.

\section{PRACTICAL ANALYSIS}

This simulation study is done using NS2. For practical analysis of proposed VHD algorithm we used below listed networks:

$$
\begin{aligned}
& \text { - WIMAX } \\
& \text { - WIFI } \\
& \text { - WLAN } \\
& \text { - GSM } \\
& \text { - LTE } \\
& \text { - UMTS }
\end{aligned}
$$

The network is composed of this all wireless networks for the evaluation of proposed work. The application considers here are audio, video and text.

Table 1: Network Scenario

\begin{tabular}{|l|l|}
\hline Number of Nodes & 10 \\
\hline Traffic Patterns & CBR (Constant Bit Rate) \\
\hline Network Size & $1000 \times 1000$ \\
\hline Max Speed & $5 \mathrm{~ms} / 10 \mathrm{~ms} / 15 \mathrm{~ms} / 20$ \\
$\mathrm{~ms}$
\end{tabular}

\begin{tabular}{|l|l|}
\hline Pause Time & $2.0 \mathrm{~s}$ \\
\hline Routing Protocol & AODV \\
\hline VHD & FR_HMM \\
\hline Application & Text/Audio/Video \\
\hline
\end{tabular}

Following figures 3, 4 and 5 are showing the achieved so far for the performance metrics such as throughput, energy consumption and packet delivery ratio (PDR) respectively. The performance of proposed VHD method is better as compared to existing method

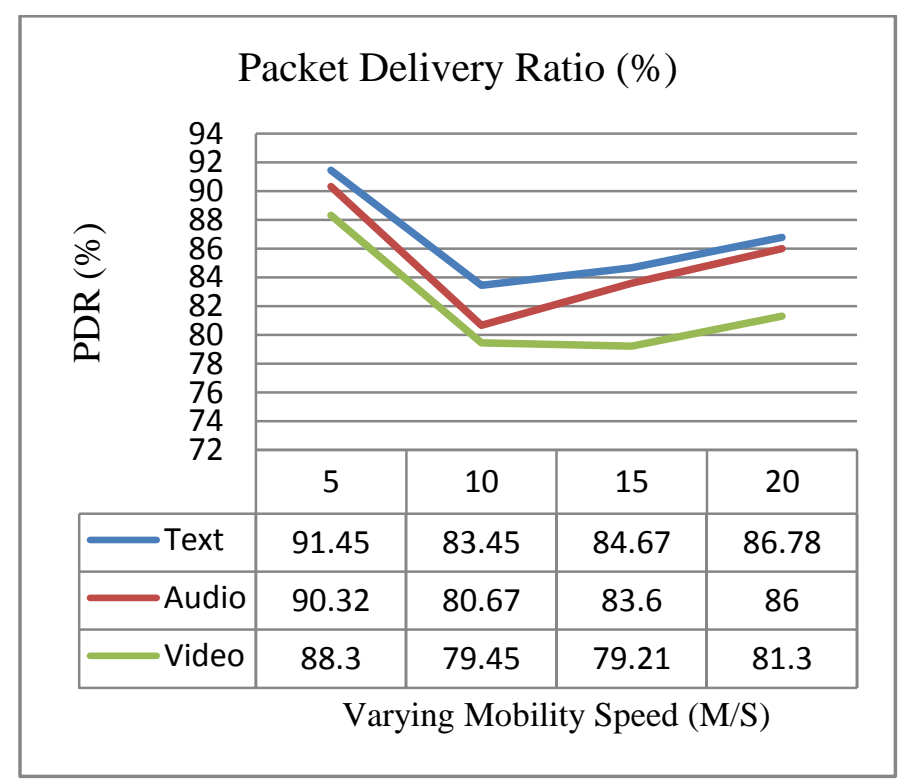

Figure 3: PDR vs. Mobility Scenarios vs. Applications

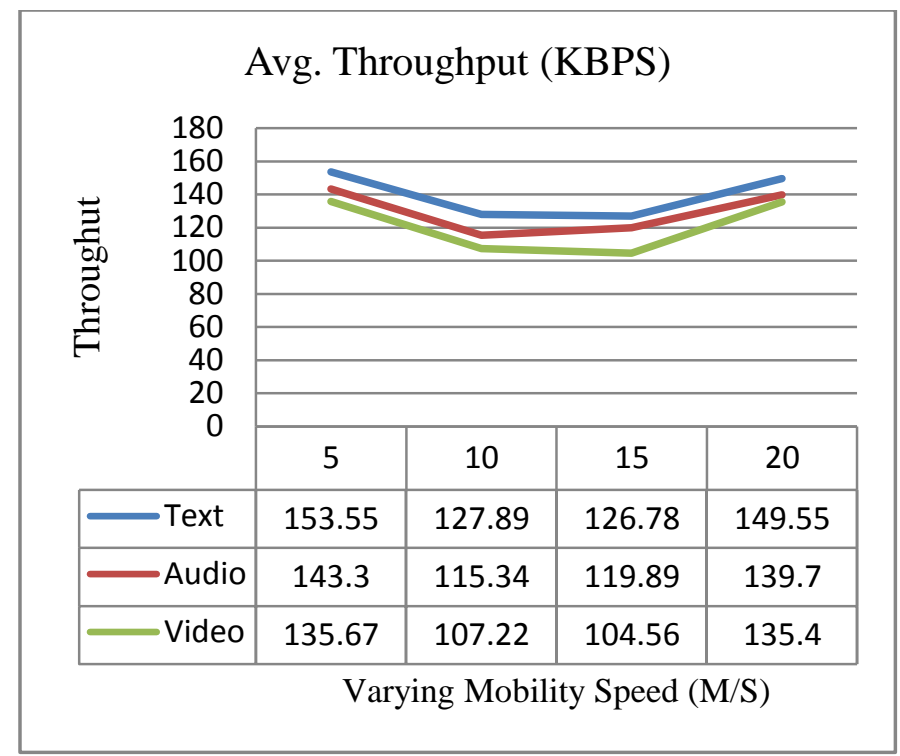

Figure 4: Average Throughput vs. Mobility Scenarios

Figures 3 and 4 are showing the performances of packet delivery ratio and average throughput respectively. From this graphs it is showing that performance of FR-HMM is better when application is Text, however still there is improvement in audio and video applications performances as compared to existing methods. 


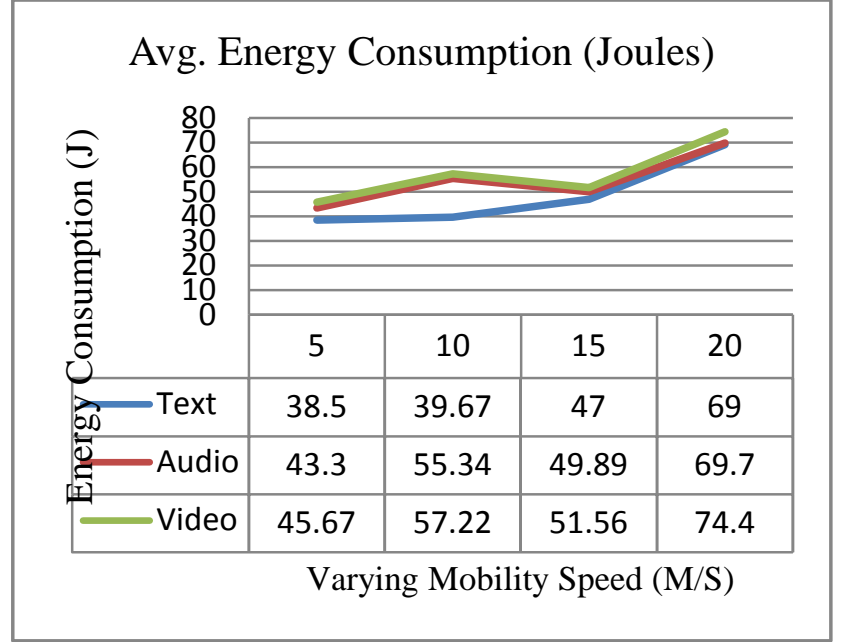

Figure 5: Energy Consumption vs. Mobility Scenarios

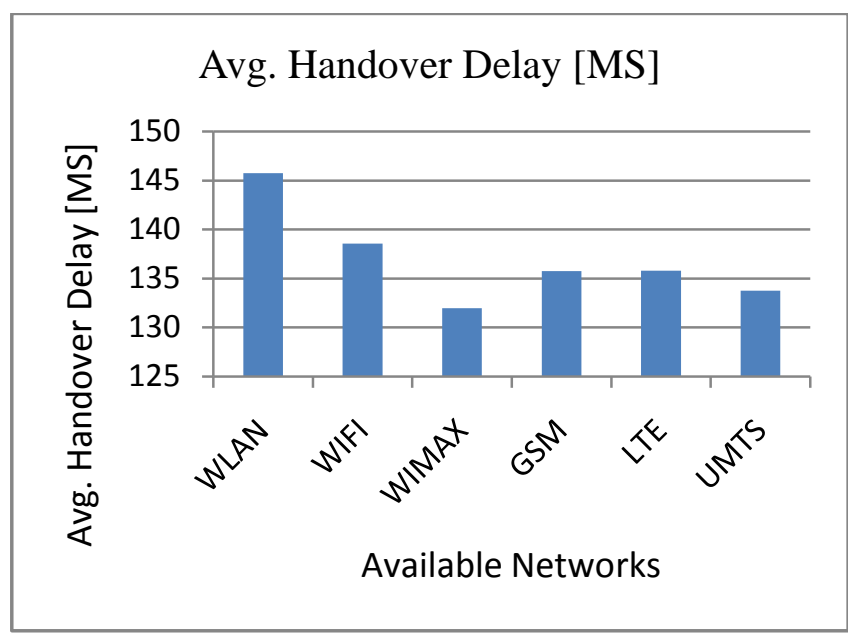

Figure 6: Handover delay Vs. Available Wireless Networks

Figure 5 and 6 evaluating the performances of energy consumption and handover delay over all networks used in simulation. In an all it is depicted that proposed method achieves better VHD performance as compared to existing methods for different mobility speed and different communication application.

\section{CONCLUSION AND FUTURE WORK}

During this paper we extended our previous study on proposed approach with different types of applications used for communication. As we presented previously, the FRHMM is based on two concepts fuzzy rules and HMM model together. A Fuzzy based HMM predictor has been used which accurately estimates the next location visited by a mobile user, given current the received signal strength values of the MNs. The home AP thus predicts the list of possible APs in the movement direction of a mobile node and sends a request to the predicted APs. The current Network Load, Strong RSS and Power consumption are estimated in each AP. These APs after calculating combined weight value, sends it back to the home AP. The home AP then selects an optimum AP for the MN to perform handover, based on the sorted weight values. The information about the selected APs, are sent to the mobile user so that the user can move to that particular AP. The performance of this method is evaluated using different kinds of networks together with varying network speed and varying type of application. For the future we further suggest to investigate this method using different network sizes and other important parameters.

\section{REFERENCES}

[1] Jian Chang, Krishna K. Venkatasubramanian, Member, IEEE, Andrew G. West, Sampath Kannan, Insup Lee, Fellow, IEEE, Boon Thau Loo, and Oleg Sokolsky, Member, IEEE, "AS-CRED: Reputation and Alert Service for Interdomain Routing", IEEE SYSTEMS JOURNAL, VOL. 7, NO. 3, SEPTEMBER 2013.

[2] Zafeiris V.E., Giakoumakis E.A., "An Agent-based Architecture for Handover Initiation and Decision in 4G networks “, June 2005.

[3] Bing H., He C., Jiang L., "Performance Analysis of Vertical Handover in a UMTS-WLAN Integrated Network “, September 2003.

[4] Aust S., Gorg C., Pampu C., “ Proactive Handover Decision for Mobile IP based on Link Layer Information “, June2010.

[5] Kassar M., Kervella B., Pujolle G., " An Intelligent Handover Management System for Future Generation Wireless Networks “, EURASIP Journal on Wireless Communications and Networking, Research Article, Laboratoire d'Informatique de Paris 6 (LIP6), University Pierre et Marie Curie (Paris6), CNRS UMR 7606. 2008.

[6] H. Chan, et al. "A utility based network selection scheme for multiple services in heterogeneous networks". International Conference on Wireless Networks, Communications and Mobile Computing. vol.2, pp. 1175 - 1180. 2005.

[7] Wei-wei Jiang, Hong-yan, Cui Qiang-jun, Van Xiaojuan, Wang Jian-Ya Chen, "A Novel ApplicationOriented Dynamic Network Selection in an Integrated UMTS and WiMAX Environment",IEEE.2008.

[8] Olga Ormond, "Economic Model for Cost Effective Network Selection Strategy in Service Oriented Heterogeneous Wireless Network Environment", IEEE.2006.

[9] Feng He, Furong Wang, "A cost sensitive best network selection scheme in heterogeneous wireless networks", 1753-1756,IEEE. 2007.

[10] F. Barcelo, Performance analysis of handoff resource allocation strategies through the state-dependent rejection scheme, IEEE Transactions on Wireless Communications 3 (3) (2004) 900-909.

[11] Elaheh Arabmakki, Sherif Rashad, Member, IEEE, \& Sadeta Krijestorac. An Algorithm for Optimizing Vertical Handoff between WLAN and Cellular Networks, Cyber Journals: Multidisciplinary Journals in Science and Technology, Journal of Selected Areas in Telecommunications (JSAT), August Edition, 2011.

[12] S. Mohanty, I.F. Akyildiz, A cross-layer (layer $2+3$ ) handoff management protocol for next-generation wireless systems, IEEE Transactions on Mobile Computing 5 (10) (2006) 1347-1360.

[13] S.Aghalya*, P.Seethalakshmi, "Prediction based Vertical Handoff Decision Algorithm in Heterogeneous Wireless Networks" IJCSET |January 2012| Vol 2, Issue 1,813-816 